\title{
Single-Collector Experiments and Modeling of Acoustically Aided Mesh Filtration
}

\author{
Michael T. Grossner \\ Case Western Reserve University \\ Donald L. Feke \\ Case Western Reserve University \\ Joanne M. Belovich \\ Cleveland State University
}

Follow this and additional works at: https://engagedscholarship.csuohio.edu/encbe_facpub

Part of the Chemical Engineering Commons

How does access to this work benefit you? Let us know!

\section{Publisher's Statement}

This is the accepted version of the following article: Grossner, M. T., Feke, D. L., , \& Belovich, J. M. (2005). Single-collector experiments and modeling of acoustically aided mesh filtration. AIChE Journal, 51(6), 1590 - 1598. doi:10.1002/aic.10423, which has been published in final form at http://onlinelibrary.wiley.com/doi/10.1002/aic.10423/abstract

\section{Original Citation}

Grossner, M. T., Feke, D. L., , \& Belovich, J. M. (2005). Single-collector experiments and modeling of acoustically aided mesh filtration. AIChE Journal, 51(6), 1590 - 1598. doi:10.1002/aic.10423

\section{Repository Citation}

Grossner, Michael T.; Feke, Donald L.; and Belovich, Joanne M., "Single-Collector Experiments and Modeling of Acoustically Aided Mesh Filtration" (2005). Chemical \& Biomedical Engineering Faculty Publications. 41.

https://engagedscholarship.csuohio.edu/encbe_facpub/41

This Article is brought to you for free and open access by the Chemical \& Biomedical Engineering Department at EngagedScholarship@CSU. It has been accepted for inclusion in Chemical \& Biomedical Engineering Faculty Publications by an authorized administrator of EngagedScholarship@CSU. For more information, please contact library.es@csuohio.edu. 


\title{
Single-Collector Experiments and Modeling of Acoustically Aided Mesh Filtration
}

\author{
Michael T. Grossner and Donald L. Feke \\ Dept. of Chemical Engineering, Case Western Reserve University, Cleveland, OH 44106 \\ Joanne M. Belovich \\ Dept. of Chemical and Biomedical Engineering, Cleveland State University, Cleveland, $\mathrm{OH} 44115$
}

\section{Introduction}

Recently, the application of acoustic fields for the processing of suspensions of small particles or living cells has been the focus of much research. Methods for particle agglomeration, manipulation, or phase separations have been reported. ${ }^{1-3} \mathrm{Bi}-$ ological applications include concentrating suspensions of cells or bacteria, filtering the suspended components from their host liquids, or enhancing characterization and detecting such species. ${ }^{4-6}$ The susceptibility of a suspended particle to an acoustic field, which is the basis for all separation and manipulation methods, is related to the density difference between the particle and the surrounding fluid as well as the difference in the speed of sound within the particle and that of its host liquid.
The focus of our interest is a particle-filtration technique that involves the application of a resonant acoustic field to a porous mesh containing pores that are generally one to two orders of magnitude larger than the particles being collected. In this case, physical screening without an acoustic field is not significant. However, with the application of an appropriate ultrasonic field, single-pass collection efficiencies up to $90 \%$ have been achieved. $^{7}$

To understand the basic phenomena that underlie this particle-filtration method, previous work has analyzed the motion of suspended particles near a small portion (a single element) of the porous mesh. This technique is a standard approach to model filtration in a complex geometry. ${ }^{8}$ Using established acoustic theory, the forces acting on particles flowing around an element of the porous mesh were modeled to predict particle paths ${ }^{9}$ in the vicinity of collection surfaces. This analysis provides insight into the particle capture mechanisms and forms a basis for a macroscopic performance model of this filtration method. However, detailed comparison of the predic- 


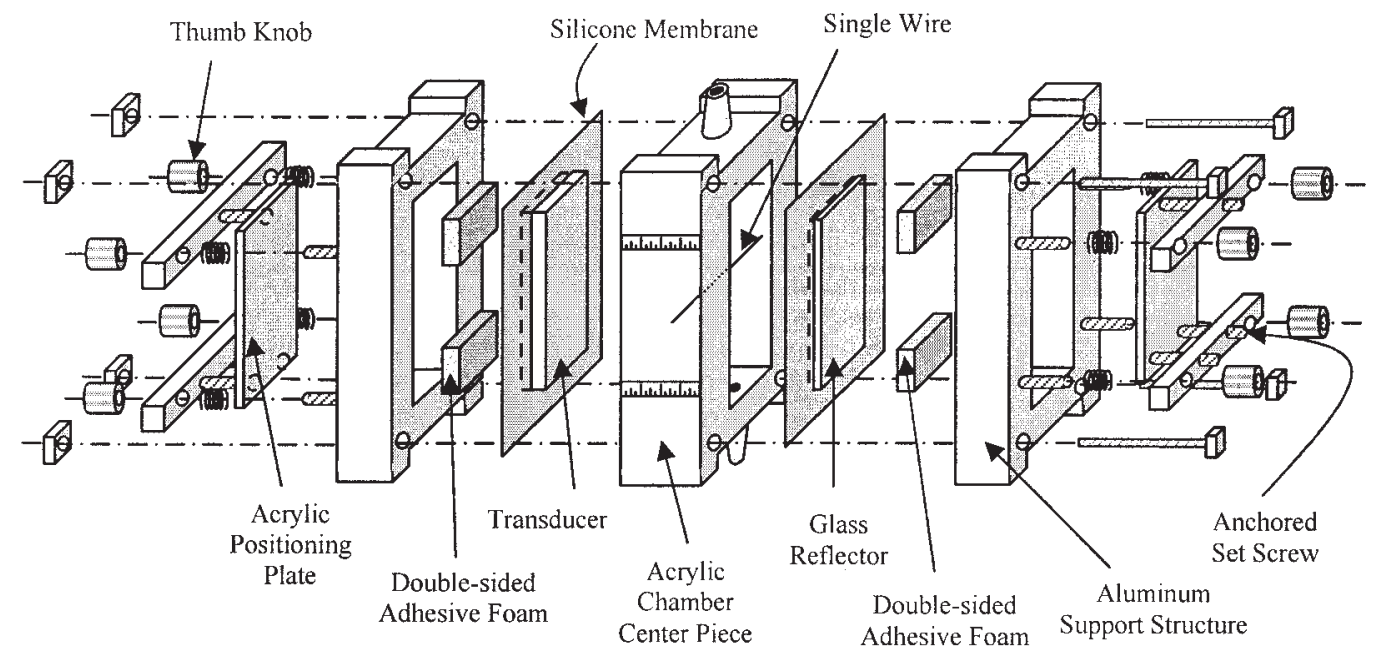

Figure 1. An expanded view of the chamber assembly.

tions of the trajectory analysis relative to experimental observation is necessary for complete verification of this model.

Experiments designed to test the validity of the singleelement trajectory-analysis model are described herein. Results of visualization experiments in which particle trajectories in the vicinity of a single cylindrical collector are presented and compared to model predictions computed using experimental conditions as model inputs.

\section{Experimental}

\section{Apparatus}

The basic design of the acoustic flow chamber was very similar to previous separation units. ${ }^{10,11}$ A rectangular piezoelectric transducer (PZT, Navy Type I, EDO Electro Ceramics Corporation, Model EC-64, $4.60 \mathrm{~cm} \times 7.75 \mathrm{~cm} \times 10.03 \mathrm{~mm}$ ) formed one wall of the chamber, whereas a glass sheet $(4.85$ $\mathrm{cm} \times 8.20 \mathrm{~cm} \times 1.04 \mathrm{~mm}$ thick) formed the opposite wall. The main body of the chamber was acrylic. A thin silicone sheet was glued around the edges of the transducer and also around the edges of the glass reflector. This allowed a seal to be made when two supporting aluminum pieces were clamped to the structure. Also, having the transducer and reflector suspended without a rigid attachment to the support structure reduced losses of acoustic energy. Adjustable knobs attached to the aluminum support structure were used to align the transducer and reflector; an exactly parallel configuration is ideal for optimum resonance conditions. The knobs pushed against an acrylic positioning plate, which was attached to the transducer (and reflector) with two pieces of foam. See Figure 1 for a schematic of the assembly.

A single stainless-steel wire fixed inside the chamber served as the collector. This particular material was chosen because of its rigidity (to reduce bowing in the flow) and its availability in small, precise diameters. The wire was passed though a hole in one side of the acrylic center piece and friction fitted inside a hole drilled $2 \mathrm{~mm}$ into the inner wall of the other side of the center piece. This configuration enabled an unobstructed view of the wire cross section looking into the chamber through the wall with the partially drilled hole. A small amount of silicone glue was placed at the end of the wire protruding from the acrylic to seal the hole.

Figure 2 depicts the overall schematic of the experimental apparatus. Feed suspension was transferred from a magnetically stirred flask to the chamber by a Masterflex peristaltic pump (model 7520-00), through 6.2-mm-diameter tubing. The flow rate was generally $0.50 \mathrm{~cm}^{3} / \mathrm{s}$ (chosen to give a linear flow rate close to $1 \mathrm{~mm} / \mathrm{s}$ inside the chamber), and passed through a small volume $\left(\sim 20 \mathrm{~cm}^{3}\right)$ hemispherical chamber to reduce flow pulsations. The suspension inside this chamber was stirred with a magnetic stirring bar. After passing through the pulse reducer, the suspension flowed into the acoustic device.

To produce a more uniform flow, a small piece of foam (nominally 10 pores/in) was placed just after the inlet inside the acoustic chamber, which thus evenly distributed the flow through the remainder of the void space in the device. After the suspension passed through the chamber, it was recycled into the stirred-feed flask.

To power the transducer in the acoustic chamber, a KrohnHite 2100A signal generator was connected in series with a 50-dB EIN 240L RF power amplifier. A Clarke-Hess 2330 Sampling V-A-W instrument was used to measure the voltage

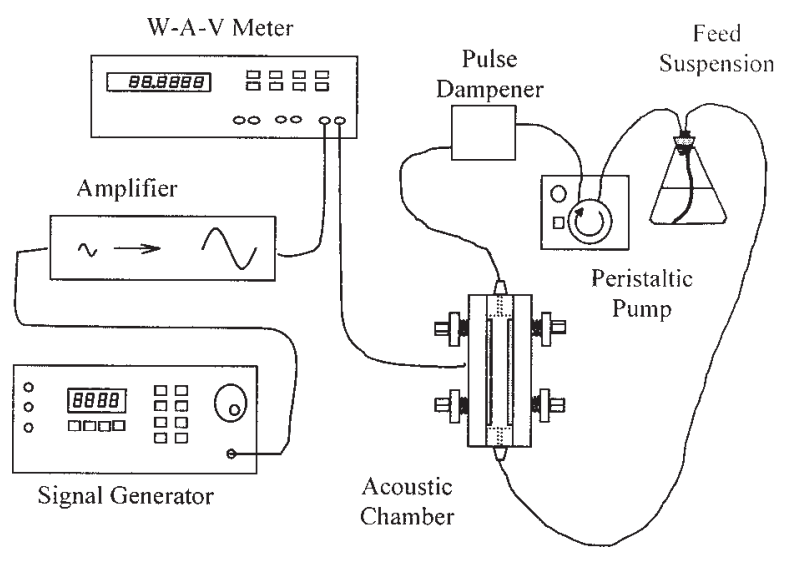

Figure 2. Experimental setup. 
across and current through the transducer. The chamber was operated at maximum power factor, indicating a strong resonance condition and minimum power loss in the system. The power factor is the cosine of the phase difference between the applied voltage and the applied current; a value of one indicates no phase difference. ${ }^{12}$

Images were recorded by a $1024 \times 1024,30 \mathrm{fps}$ UNIQ UP-930 digital video camera with a $12.7-\mathrm{mm}$ progressive scan CCD sensor. To image an area of $5 \times 5 \mathrm{~mm}$ with this camera, a Mirco Nikkor AF 60/2.8 lens was used, along with a set of spacers and a C-mount to F-mount adapter. The camera was mounted to a digitally controlled XYZ stage capable of measuring adjustments in position to $0.1 \mu \mathrm{m}$.

A 40-W halogen desk lamp was the illumination source for general viewing of the chamber, particularly during alignment of the transducer and reflector. For particle imaging, however, a Power Technology 532-nm, 10-mW laser (model LCM-T-11 CCS) was used. The laser light passed through a 5-mm-diameter, horizontally oriented glass rod, which created a vertical "light sheet" or laser plane. This plane passed through the glass reflector to illuminate a plane inside the chamber that had a normal along the wire axis.

Images from the camera were directly recorded into computer memory using an Epix Inc. PIXCI D2X interface card. The images were stored as $1024 \times 1024$, 10-bit grayscale TIFF files. To capture images, a software program (XCAP Interactive Image Analysis 2.2, also supplied by Epix Inc.) was run on an $800-\mathrm{MHz}$ Pentium III using Windows 2000. Besides controlling the capture and storage of the particle images, this program has tracking-analysis tools that were used.

\section{Procedures}

To begin a particle-tracking experiment, a $0.4 \mathrm{mg} / \mathrm{cm}^{3}$ suspension of polystyrene particles (54- $\mu \mathrm{m}$ mean diameter) was prepared using filtered (to $0.2 \mu \mathrm{m}$ ), deionized water. This particular concentration was chosen because it gave the best visibility of individual particles, while maximizing the total amount of visible particles in trial runs. The suspension was degassed with a simple vacuum pump to prevent any gas bubbles from interfering with particle visualization.

The acoustic chamber was assembled and connected to the flow system and the supporting electronics. After the fluid flow was established, the transducer was aligned to be parallel to the reflector through an iterative process. The distance between the transducer and glass reflector was measured and adjusted at each of the four corners using translation of the XYZ stage on which the camera was mounted. This process was continued until the measurements were within $0.1 \mathrm{~mm}$ of each other. A normal value for this spacing was around $9 \mathrm{~mm}$. Also, the transducer and reflector were measured to be exactly vertical, to $0.1 \mathrm{~mm}$, to ensure that the wire axis was perpendicular to the acoustic field.

Once the alignment was complete, the camera was positioned to view the wire in the center of the image; the wire axis was perpendicular to the image plane so that the wire appeared as a disc. The laser was switched on and the camera was focused on the light plane, which impinged on the midpoint of the wire length.

To find a resonant ultrasonic frequency, the transducer was powered with a $150-\mathrm{mV}$ sine wave from the signal generator, resulting in the application of 1.6-W rms across the transducer electrodes. The experiments were performed at this power level because a higher level caused an unacceptable amount of buoyancy flow (see below) and lower power did not produce a meaningful acoustic response of the particles.

The frequency was varied manually from a starting point of the calculated optimum resonant frequency of the chamber. ${ }^{13}$ Once a frequency was found that maximized the power factor, the power to the transducer was disconnected. Because operation of the transducer results in generation of a small amount of heat, the transducer was operated for only brief periods to minimize the impact of buoyancy-driven flow.

The approach speed of the fluid was measured by focusing the camera at a point far away from the wire and measuring particle velocities (with no active acoustic field). Also at this point, the acoustic field was activated to measure the position of the pressure nodal planes, assumed to be the locations where the particles were aligned.

Video was recorded digitally at 10 frames/s for a period of $12 \mathrm{~s}$ at a time. The linear flow rate in the chamber was about 0.5 $\mathrm{mm} / \mathrm{s}$, so images of the same particle would nominally be spaced $0.05 \mathrm{~mm}$ from frame to frame. This has proved to be sufficient for particle-tracking analysis. At 2-3 s into the recording period, the sound field was activated. The particles responded to the acoustic field, establishing a steady trajectory pattern in $<1 \mathrm{~s}$. After about 6-8 s, heat from the transducer surface caused buoyancy-driven flow that disrupted the flow profile.

After performing image-conditioning procedures, the XCAP Interactive Image Analysis software package can be used to report particle-position data. Certain limitations of this analysis, arising from either the experiment or the software package, were immediately evident. For example, the software reports particle trajectories only if the particle is present in each and every frame that is analyzed. Because the wire itself causes a shadow in the laser sheet, particle trajectories that cross the shadow could not be analyzed. Also, particles that move out of the illuminated sheet could not be analyzed.

\section{Single-Collector Trajectory Calculations}

Particle paths in the vicinity of the collector are solved from a set of differential equations that reflect a balance of forces acting on the suspended particles. A detailed discussion of the particle trajectory model is available in Gupta and Feke. ${ }^{10}$ When acoustic and hydrodynamic forces are considered, two dimensionless lumped parameters govern the particle paths. The Gor'kov number (Gk)

$$
\mathrm{Gk}=\frac{R_{p}^{2} k E_{a c}}{\mu U_{f}}
$$

characterizes the ratio of acoustic forces to hydrodynamic drag forces on the particles, whereas the Reynolds number (Re), based on the cylinder diameter

$$
\operatorname{Re}_{c}=\frac{2 U_{f} R_{c} \rho_{f}}{\mu}
$$


Table 1. Physical Properties and Parameters Used in the Single-Collector Experiments

\begin{tabular}{ll}
\hline Acoustic field & \\
Energy density $\left(E_{a c}\right)$ & $0.25 \mathrm{~J} / \mathrm{m}^{3}$ \\
Frequency $(\omega / 2 \pi)$ & $680.2 \mathrm{kHz}$ \\
Fluid: pure water & \\
Bulk velocity $\left(U_{f}\right)$ & $0.65 \mathrm{~mm} / \mathrm{s}$ \\
$\quad$ Viscosity $(\mu)$ & $0.001 \mathrm{~kg} / \mathrm{m}^{-1} \mathrm{~s}^{-1}$ \\
Density $(f)$ & $1000 \mathrm{~kg} / \mathrm{m}^{3}$ \\
Longitudinal sound speed $\left(c_{f}\right)$ & $1480 \mathrm{~m} / \mathrm{s}$ \\
Cylindrical collector: stainless steel & \\
Radius $\left(R_{c}\right)$ & $0.3 \mathrm{~mm}$ \\
Density $(c)$ & $7900 \mathrm{~kg} / \mathrm{m}^{3}$ \\
Longitudinal sound speed $\left(c_{1}\right)$ & $5790 \mathrm{~m} / \mathrm{s}$ \\
Shear sound speed $\left(c_{2}\right)$ & $3100 \mathrm{~m} / \mathrm{s}$ \\
Particles: polystyrene & \\
Radius $\left(R_{p}\right)$ & $27 \mu \mathrm{m}$ \\
Density $\left({ }_{p}\right)$ & $1050 \mathrm{~kg} / \mathrm{m}^{3}$ \\
\hline
\end{tabular}

characterizes the flow strength. The radius of the particle and the collector (cylindrical element) are $R_{p}$ and $R_{c}$, respectively. The fluid velocity far upstream from the collector is $U_{f}$; the fluid has viscosity $\mu$ and density $f$. The acoustic energy density and wavenumber are represented by $E_{a c}$ and $k$, respectively. The acoustic force acting on the particles depends on the position of the cylinder relative to the incident acoustic field, given that acoustic forces are sensitive to both the incident field and that scattered from the cylinder.

Including the effects of buoyancy forces results in a third dimensionless parameter, the Archimedes number (Ar), that affects particle trajectories

$$
\operatorname{Ar} \frac{2 R_{p}^{2}\left({ }_{p}-{ }_{f}\right) g}{9 \mu U_{f}}
$$

Values of the physical parameters used for modeling experimental conditions are shown in Table 1. The energy density inside the chamber was calculated using an independent transmission model of a resonant ultrasonic wave traveling through a layered system. ${ }^{13}$ This energy density model included attenuation and had been verified against experimental measurement within a similar chamber. ${ }^{13}$ For the experimental conditions, $\operatorname{Re}_{c}=0.39, \mathrm{Gk}=0.81$, and $\mathrm{Ar}=0.12$.

\section{Comparison metric}

To compare a particle trajectory observed in an experiment to one simulated in the model, the area between a predicted and an observed particle path (in a two-dimensional projection of both paths) was computed. This comparison is not intended to label a predicted trajectory as a good or bad fit to a measured trajectory in an absolute sense, but rather to give an indication of a better or worse fit when adjusting model parameters. When the area between the model and experimental trajectories decreases, it is assumed that the model is providing a better reproduction of the experimental system.

\section{Results and Discussion}

\section{Experiments with no acoustic forces}

Preliminary experiments were done to establish that the trajectory model adequately described the particle motion in the absence of acoustic forces, and that the experimental technique generated a steady, uniform motion in the vicinity of the cylinder. Plots of two sample particle paths are shown in Figures 3 and 4. In these figures, the circle represents the cross section of the cylindrical collector. The closely spaced lighter points, which appear as a continuous line, represent the predicted path of the particle. The large points are data from the particle tracking experiments. The units in the plots are dimensionless, with distances scaled to the radius of the wire (1 radius $=0.3 \mathrm{~mm}$ ).

Model predictions closely matched experimentally observed trajectories, although the observed data seemed slightly shifted in the downstream flow direction. This discrepancy may arise from a slight inaccuracy in the coordinate system definition for that particular tracking experiment.

\section{Experiments involving acoustic fields}

Comparison of Experimental Results with Pure Predictions of the Trajectory Model. Three of these sets of observed and predicted trajectories are shown in Figures 5-7 with one experimentally measured particle path per plot. In these figures, the parallel, dashed lines indicate the pressure nodes of the incident acoustic wave relative to the position of the collector. The predicted trajectories were computed based on the model parameters shown in Table 1. Because the transducer generating the acoustic field is positioned off to the bottom of the frames depicted in these figures, one may expect that the cylindrical collector interferes with the incident field in its vicinity. Thus, the prediction of the acoustic forces was based on the magnitude and geometry of the reflected acoustic field.

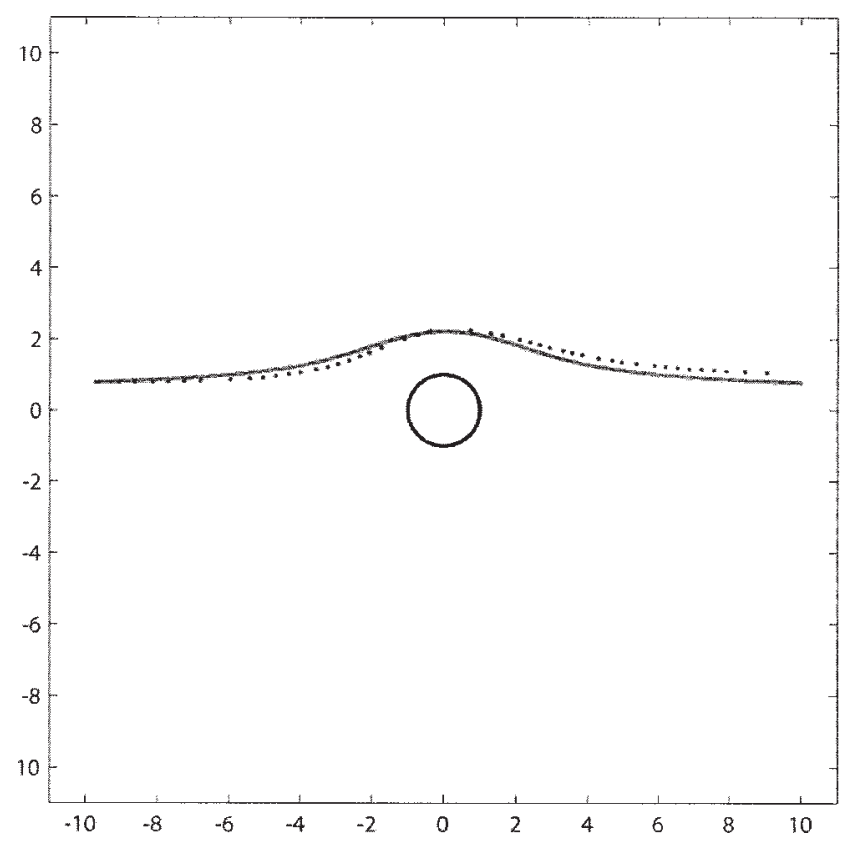

Figure 3. A plot of an experimentally measured particle trajectory (points) along with a simulated one (line) for the case of no acoustic forces.

The circle in the center is the cross section of the cylindrical collector. The units of the plot are dimensionless, scaled to the radius of the collector $(1$ radius $=0.3 \mathrm{~mm})$. The model prediction well matches the experimental data (points). 


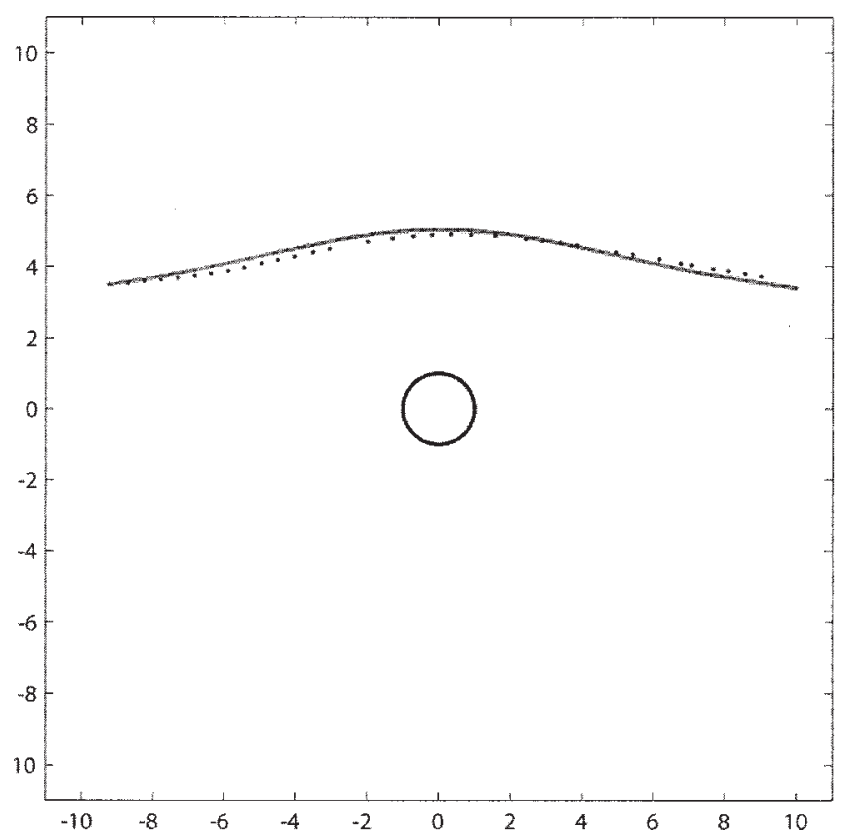

Figure 4. Another particle trajectory in the absence of acoustic forces.

The particle in this plot travels in a path farther from the collector than the one depicted in Figure 3.

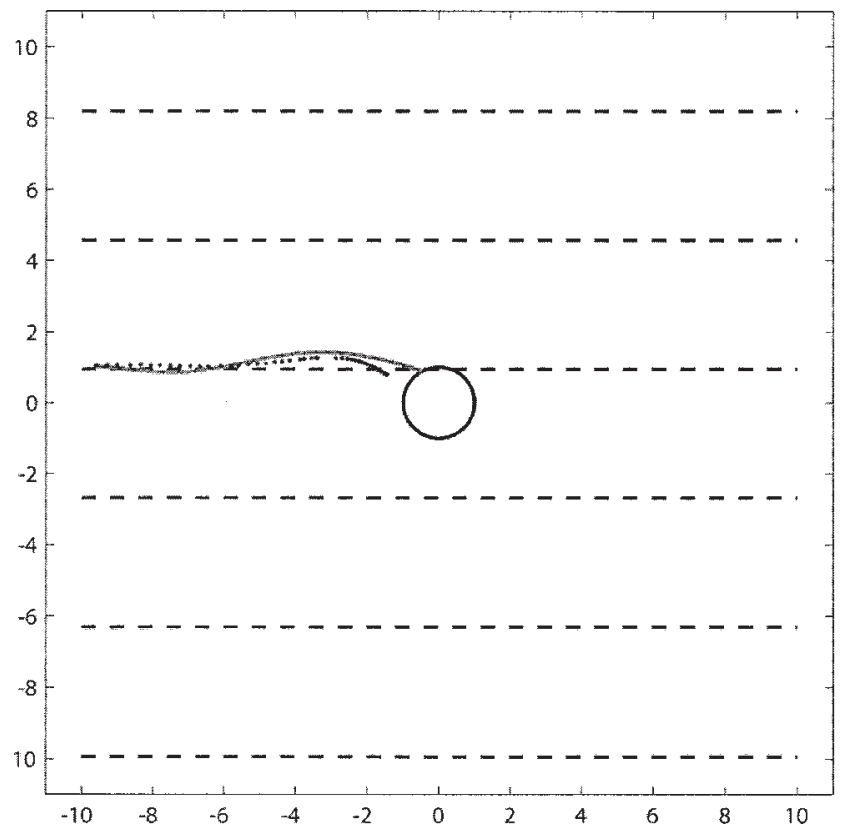

Figure 5. Predicted and observed trajectories in an experiment with applied acoustic forces.

The parallel, dashed lines indicate the pressure nodes of the incident plane wave. For ease in identification, the experimental trajectory shown here is referred to in the text as trajectory

A. The simulated particle trajectory follows close to the path observed in the experiment. In the experiment, the particle does indeed collide with and stick to the cylinder. This is consistent with the model prediction. The goodness-of-fit metric (the area between the predicted and observed trajectories) is 1.16 .

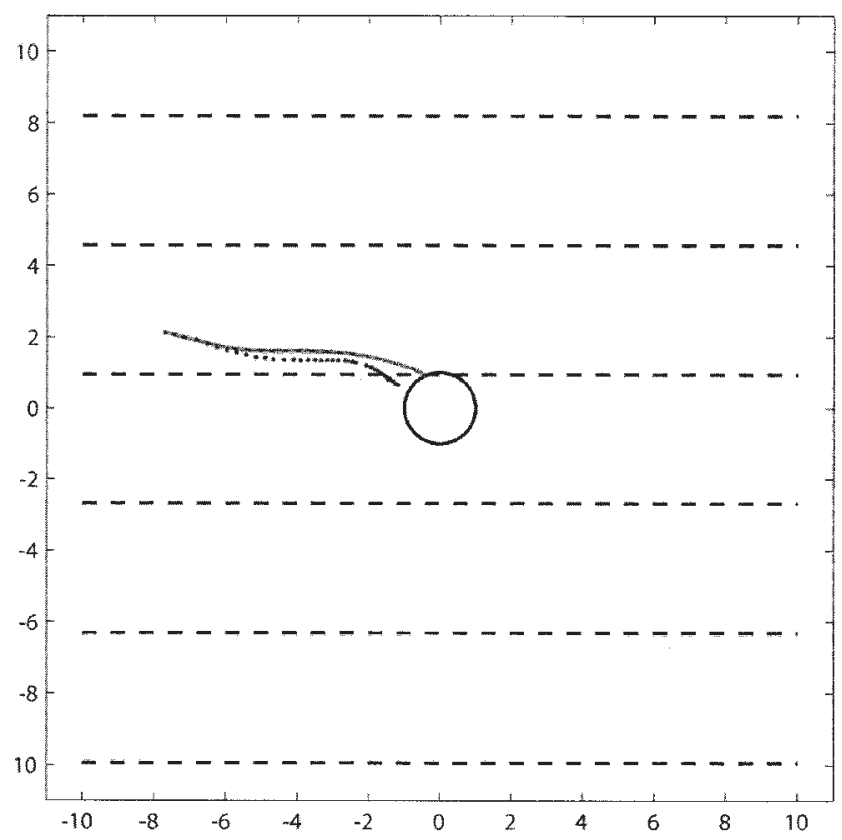

Figure 6. Another experimentally determined particle trajectory, referred to as B in the text, shown with its simulated counterpart.

As in the case for A, here the trajectory is predicted to collide with the cylinder. The goodness-of-fit metric is 1.14.

For ease of discussion, the observed trajectories depicted in Figures 5-7 shall be referred as A, B, and C, respectively. These three experimental trajectories were chosen to illustrate

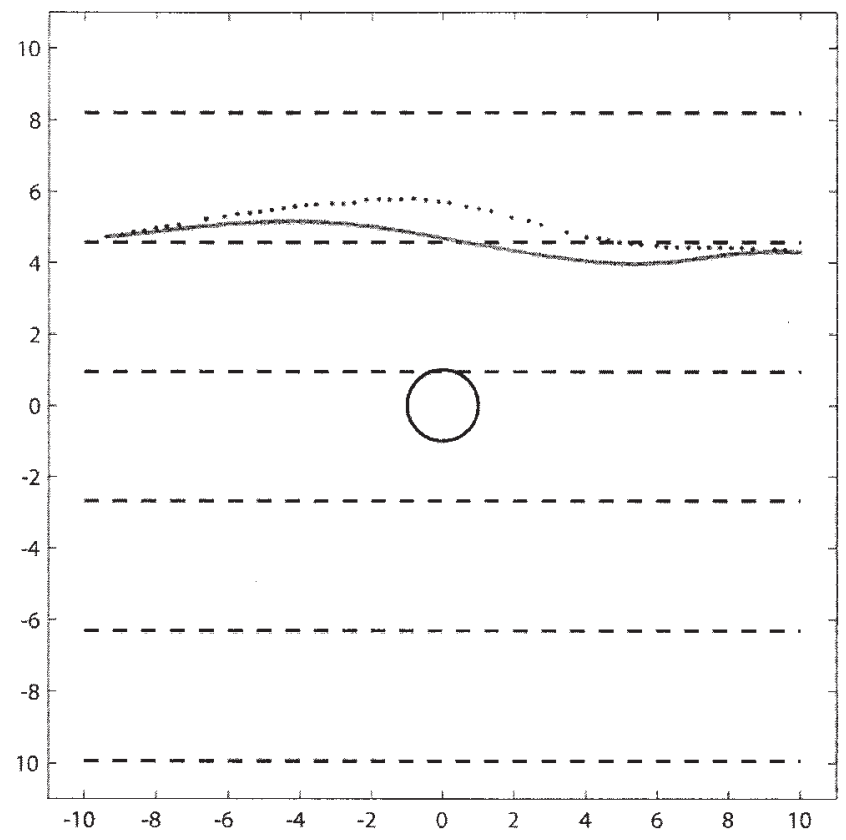

Figure 7. This experimental trajectory, referred to as C in the text, remains at least five radii from the collector.

The curvature of the model trajectory is similar to that observed in the experiments and the goodness-of fit metric between the two trajectories is 9.63 . 
Table 2. Parameters and Results of the Comparisons between Model and Experiment*

\begin{tabular}{cccccccc}
\hline & $\begin{array}{c}\text { Starting } \\
\text { Position }\end{array}$ & & \multicolumn{2}{c}{$\begin{array}{c}\text { Pure } \\
\text { Prediction }\end{array}$} & & \multicolumn{2}{c}{ Optimized Fit } \\
\cline { 2 - 3 } $\begin{array}{c}\text { Experimental } \\
\text { Trajectory }\end{array}$ & $y$ & & Gk & $\begin{array}{c}\text { Area } \\
\text { Metric }\end{array}$ & & Gk & $\begin{array}{c}\text { Area } \\
\text { Metric }\end{array}$ \\
\hline A (experiment 1) & 1.0 & & 0.81 & 1.16 & & 1.10 & 1.00 \\
B (experiment 1) & 2.1 & & 0.81 & 1.14 & & 1.04 & 0.46 \\
C (experiment 2) & 4.7 & & 0.81 & 9.63 & & 0.29 & 1.72 \\
D (experiment 1) & 3.6 & & 0.81 & 11.0 & & 0.065 & 5.38 \\
E (experiment 2) & 0.0 & & 0.81 & 1.69 & & 1.30 & 1.42 \\
\hline
\end{tabular}

*The starting position is the value of $y$ (that is, the vertical distance from the axis of the cylinder) in the plot at the initial point of the experimentally measured particle trajectory. Both Gk and the area metric are dimensionless.

typical particle behaviors in the vicinity of the collector. In both $\mathrm{A}$ and $\mathrm{B}$, the incoming particle, although originating at a unique location, collides with and sticks to the same point on the collector. The path of $\mathrm{C}$ remains roughly five radii from the collector at its closest approach.

The model calculations match the experimental trajectories quite well, that is, the solid line follows the same general path as the experimental points plotted in Figures 5-7. Also, trajectories $\mathrm{A}$ and $\mathrm{B}$ are predicted to terminate at the collector surface, consistent with the experimental results. Because these are pure model predictions without any adjustment to model input parameters, the agreement with experimental trajectories is quite good.

Effect of Nonuniform Acoustic Energy Density. The predictions of the particle trajectory model are calculated on the basis of a single value for Gk to describe the relative strength of acoustic forces. This presumes that all individual parameters contained within Gk are known and constant for all experimental trajectories. However, because of attenuation and scattering of the acoustic field, the acoustic energy density is likely not to be uniform in the vicinity of the cylindrical collector. Thus, accurate prediction of particle trajectories would require knowledge of the spatial variation of Gk along a particle path, which is not known a priori. Thus, the value of Gk used within the model predictions should be viewed as a chamber-averaged value.

Adjustment of the value of Gk allows the model to more accurately predict the experimental particle trajectories as gauged by the area metric. Selection of $\mathrm{Gk}=1.10,1.04$, and 0.29 optimizes the fits for A, B, and C, respectively. The area metrics for the original and optimized cases are presented in Table 2. Plots of these optimized fits are shown in Figures 8-10. The adjusted values of Gk are well within an order of magnitude of the pure prediction based on the acoustic energy density obtained from the multilayer transmission model. ${ }^{13}$

One explanation for the difference in the optimized Gk values is that trajectories $\mathrm{A}$ and $\mathrm{B}$ were taken from a single experiment, whereas $\mathrm{C}$ was taken from a different one. Because optimization of the fit for A and B resulted in nearly the same corrected energy density, it is possible that a systematic inconsistency, such as temperature fluctuation could have changed the energy density (by altering the resonant frequency slightly) inside the chamber. Another explanation is that the singlecollector model does not properly predict the decreasing effectiveness of the acoustic field as the position of the particle is

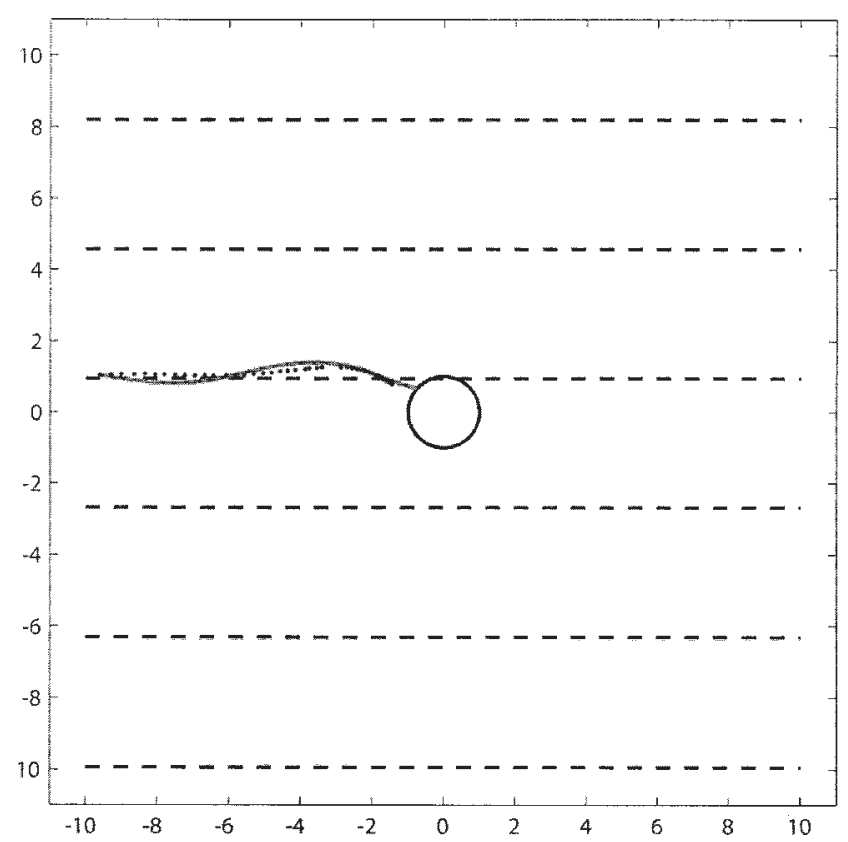

Figure 8. An improved fit for trajectory A was found by increasing Gk from 0.81 to 1.10 , which decreased the area between trajectories to 1.00 .

farther from the cylinder. Trajectories A and B passed much closer to the collector than trajectory $\mathrm{C}$.

To determine which of these scenarios is more likely, a trajectory from the same experiment as A and B, but farther from the wire, was examined. This trajectory (hereafter referred to as D) is shown in Figure 11, along with the simulated trajectory using the original Gk of 0.81 , and again in Figure 12

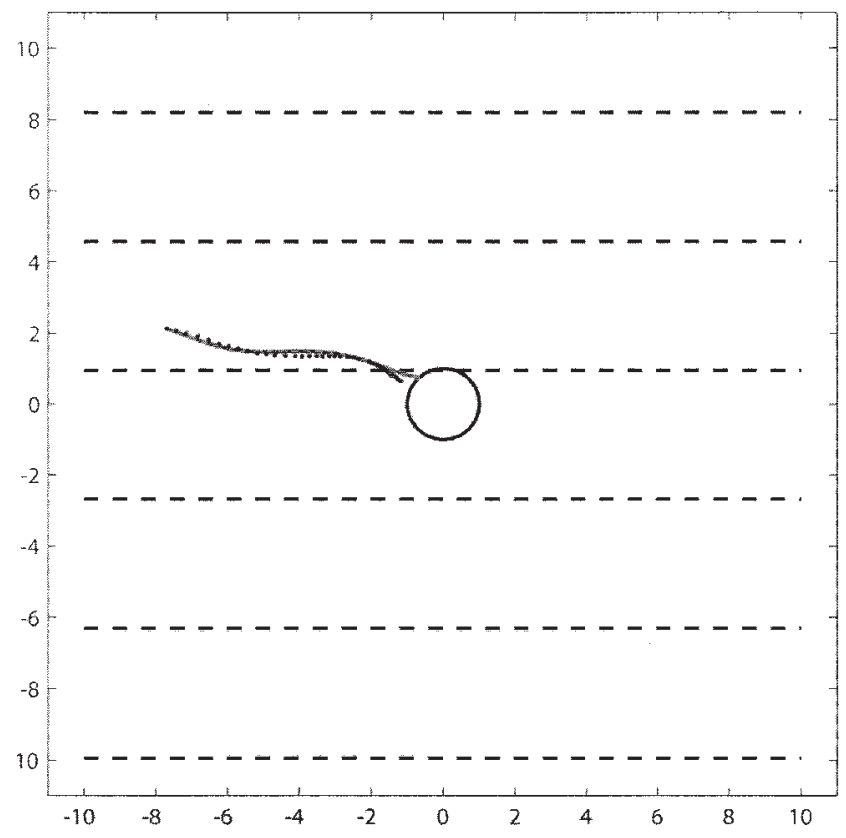

Figure 9. An improved fit for trajectory $B$ was found by increasing Gk from 0.81 to 1.04 , which decreased the area metric to 0.46 . 


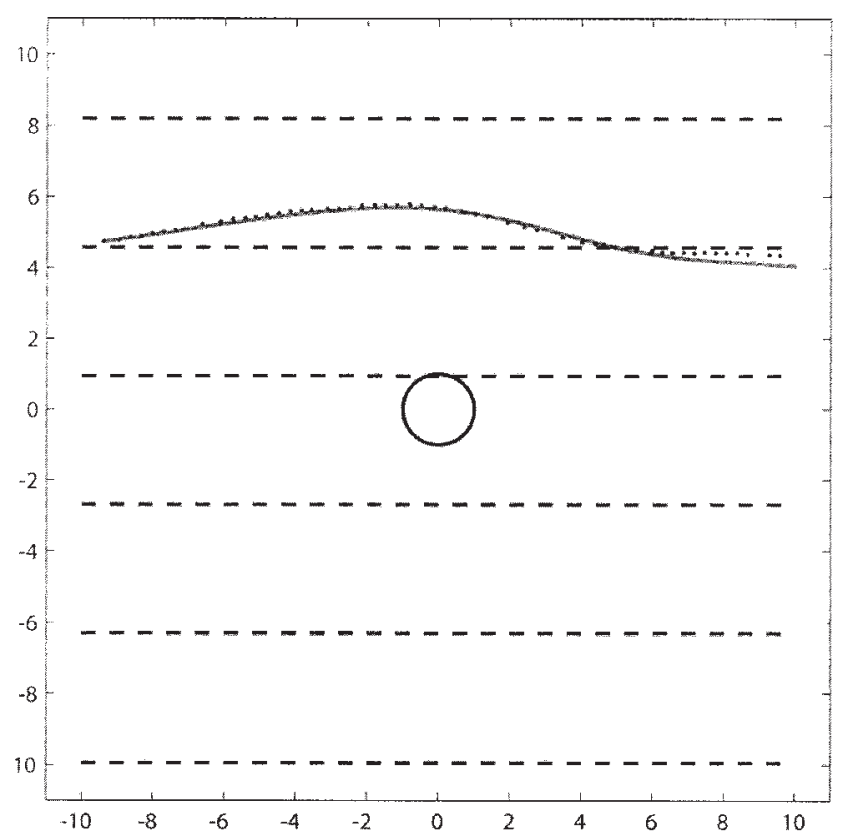

Figure 10. An improved fit for trajectory $\mathrm{C}$ was found by decreasing Gk from 0.81 to 0.29 , which decreased the area metric to 1.72 .

with the simulated trajectory using a best-fit value for Gk of 0.065 . This result follows the same trend as that of trajectory $\mathrm{C}$, although in $\mathrm{C}$, the value of Gk was decreased to only 0.29 .

To make another comparison, a trajectory from the same experiment as $\mathrm{C}$, but that passed closer to the collector, was

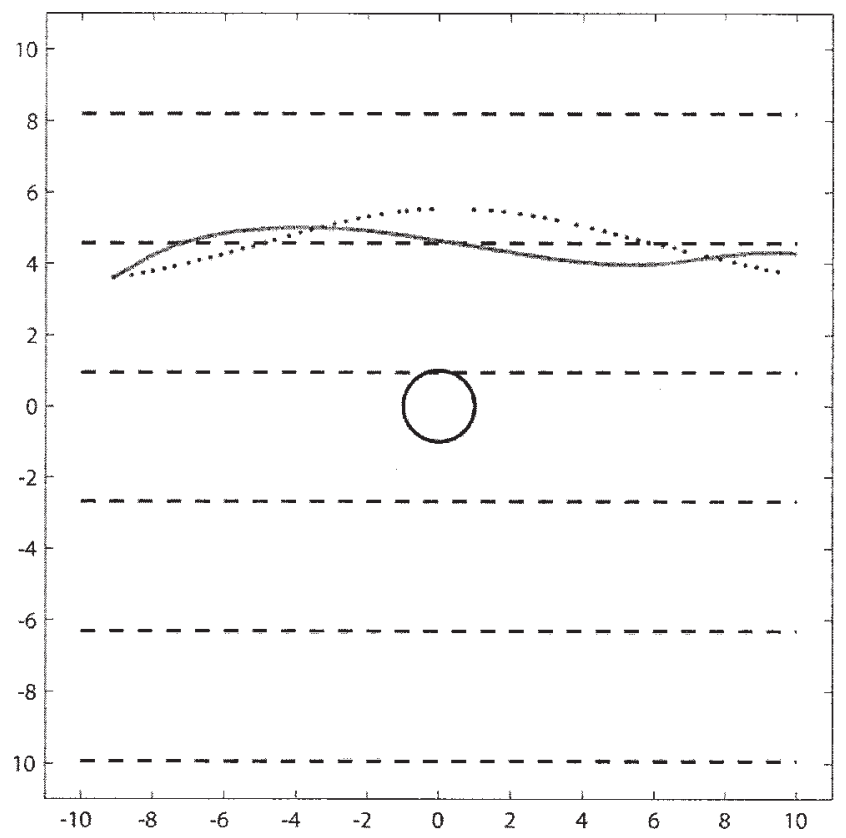

Figure 11. An additional trajectory (referred to as $D$ in the text) from the same experiment as $A$ and $B$, but in this case the particle is farther from the collector.

The area metric for this case of $\mathrm{Gk}=0.81$ is 11.0 .

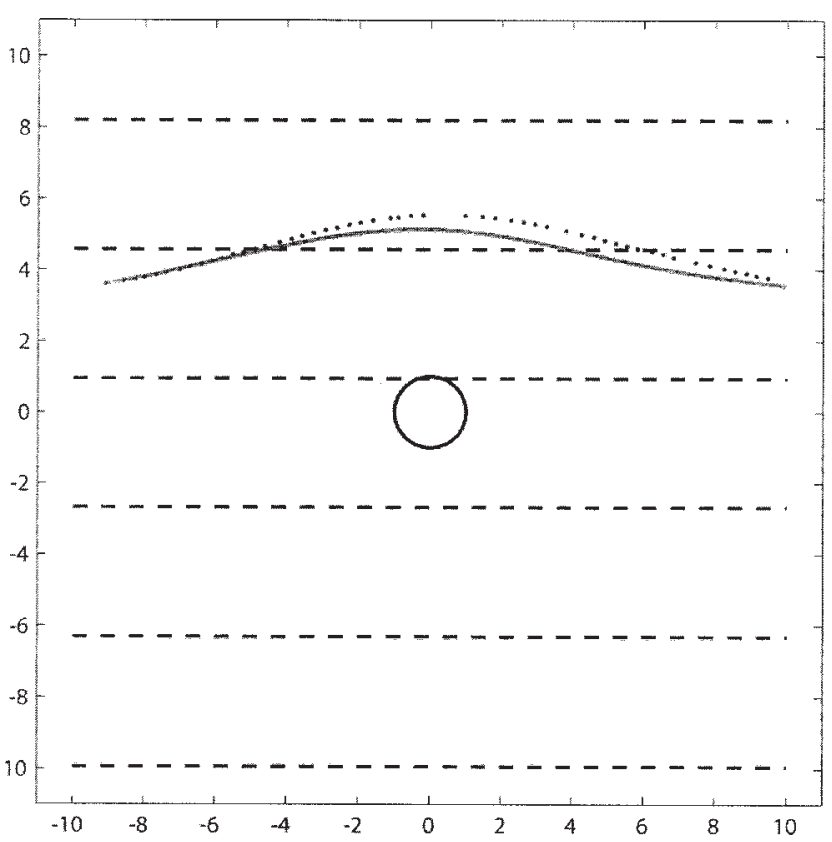

Figure 12. Trajectory $D$ is shown again with a best fit of Gk $=0.065$, decreased significantly from the original parameter value.

The area metric is 5.38 .

analyzed. The plots of this trajectory (referred to as E) with simulated particle paths are depicted in Figures 13 and 14. The best fit for $\mathrm{E}$ was found by increasing the value of Gk to 1.30. Again, this follows the same trend as that for A and B, that is,

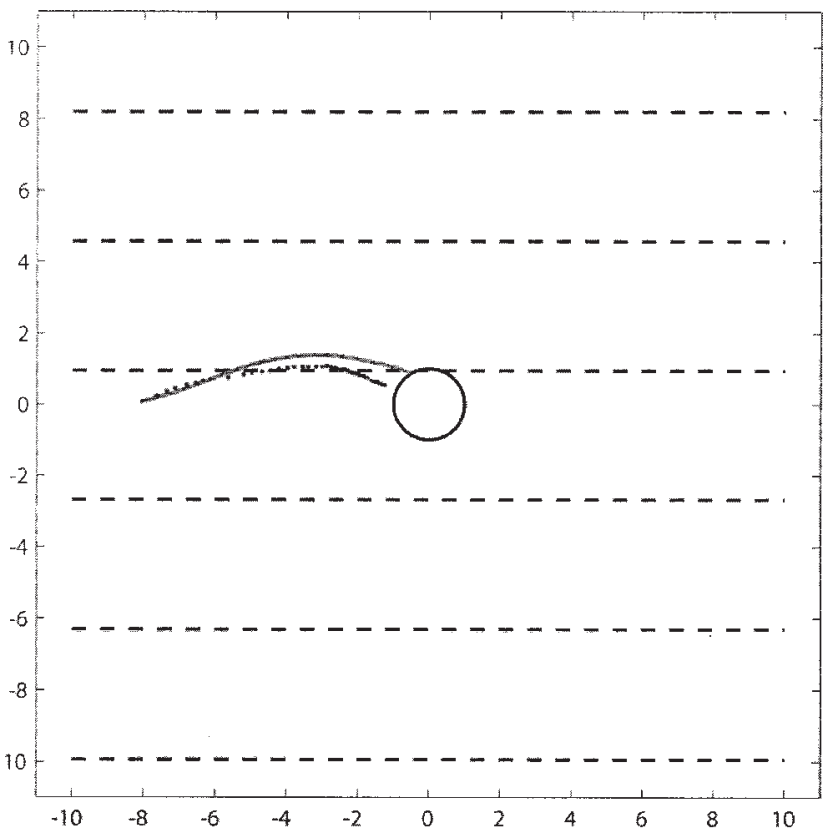

Figure 13. This experimental trajectory (E) was taken from the same experiment as $C$, but passes close to the collector.

This is the no-parameter-adjustment fit, with Gk $=0.81$. The area metric is 1.69 . 


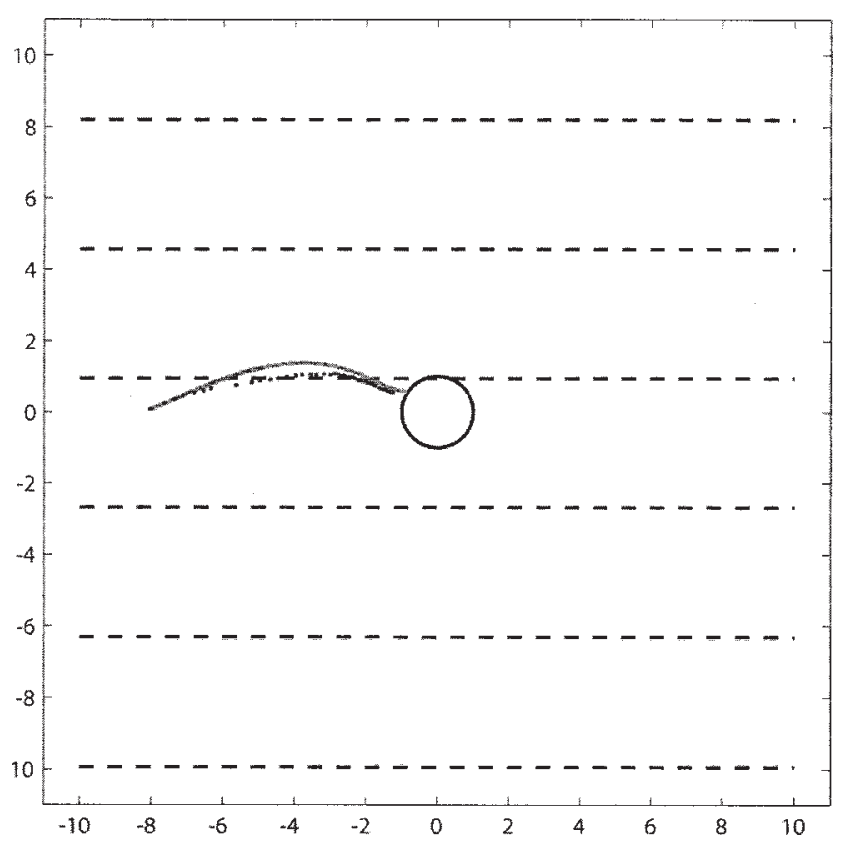

Figure 14. Increasing Gk to 1.30 for the simulated trajectory decreases the area metric to $\mathbf{1 . 4 2}$.

for these trajectories near the collector, the effect of the acoustic field in the model needed to be increased for it to match the experiment.

A summary of the parameter values and areas between trajectories is given in Table 2. From the preceding analysis, it is likely that the model does not adequately account for the attenuation of the acoustic field as the distance from the collector increases. The modeled effect of the acoustic field needed to be decreased significantly in the two trajectories that were far from the collector (C and D) to fit the experimental particle paths, but also needed to be increased by as much as $60 \%$ to match $\mathrm{A}, \mathrm{B}$, and $\mathrm{E}$, which traveled more closely to the cylinder.

\section{Discussion of error}

The most significant source of uncertainty in the prediction of particle trajectories is the accuracy of the acoustic energy density estimated using the multilayer resonance model. The multilayer model has been found to be quite accurate when the experimental chamber is tuned to be highly efficient (the system operates at maximum reflected power). ${ }^{13}$ However, because the experiments in the current study were limited to a short acoustic field activation periods, it is possible that the chamber was not operated at peak efficiency. Slight changes in temperature associated with the repeated application of power to the transducer could have affected the optimum resonance conditions.

Another source of error is associated with the nonuniform size of the polystyrene particles used in the experiments. Diameters ranged from 37 to $92 \mu \mathrm{m}$ with $80 \%$ of the particles having a diameter between 47 and $72 \mu \mathrm{m}$. Because Gk is sensitive to the square of the particle size, there could be more than a factor-of-2 uncertainty in the actual Gk for a given experiment, even if the acoustic energy density was known precisely. For the ends of the $80 \%$ particle size range mentioned above, Gk would range from 0.61 to 1.44 using the nominal values for the acoustic field and fluid properties. This effect could explain part of the variation in the reported optimized Gk, described in the previous section.

Comparatively, the experimental error in determining the particle position measurement is quite low. The $1024 \times 1024$ pixel size of the captured video frame was calibrated precisely with a 1/64-in. scale to give 182.4 pixels per mm or $5.482 \mu \mathrm{m}$ per pixel. Particle position could be measured to within an error of a few pixels, and thus the error in each spatial measurement was on the order of tens of microns.

It is also possible that particle-cylinder hydrodynamic interactions, not accounted for in the model, play a role for the trajectories that pass near the collector. Additionally, buoyancy-driven flows stemming from nonuniform heating of the fluid may have influenced the particle trajectories.

\section{Conclusions}

The objective of this work was to validate a previously reported trajectory model ${ }^{10}$ for the motion of particles in the vicinity of a cylindrical collector while being subjected to acoustic and flow forces. Verification was accomplished by designing an experimental system to visualize the interaction between particles and a cylinder that was subjected to a resonating ultrasonic field. A wire was suspended inside an acrylic chamber such that a digital video camera could have a visual axis parallel to its length, that is, the cross section of the wire appeared as a circle in the image plane. Particle-tracking techniques were used to analyze the images and record the position of particles as the fluid flowed around the wire in the presence of an acoustic field. These trajectories were compared with those determined by the single-collector simulation.

Particle capture by the cylinder was observed in the experiment and the experimental trajectories matched simulations without any model parameter adjustment. All of the parameters of the model were taken from actual measurements of the experimental system except the energy density of the acoustic field, which was estimated based on chamber dimensions and properties and applied power using a multilayer model. ${ }^{13}$ The fit between the single-collector model and experimental trajectories was further improved by adjustments in Gk, which represents the relative intensity of acoustic forces to flow forces. It was found that near the cylinder, acoustic forces predicted by the single-collector model were slightly higher in the experiment. At distances a few radii away from the cylinder, the model significantly overestimated the acoustic forces present.

One of the assumptions of the single-collector model was that the applied acoustic field is uniform in the chamber. Cross-correlation analysis of experimental particle images indicated that this may not be the case; the intensity of the acoustic field may have been smaller farther from the face of the transducer, that is, where the model overestimated the acoustic forces involved. This is consistent with the notion that there will be some attenuation in the sound field.

The results of this model-experiment comparison were favorable, that is, the model closely tracks the particle paths. Thus, the trajectory model can be used as the basis for analyz- 
ing the performance characteristics of acoustically aided meshfiltration processes.

\section{Acknowledgments}

This work was supported by NASA though Grant NAG8-1854.

\section{Literature Cited}

1. Hertz HM. Standing-wave acoustic trap for nonintrusive positioning of microparticles. J Appl Phys. 1995;78:4845-4849.

2. Groschl M, Burger W, Handl B, Doblhoff-Dier O, Gaida T, Schmatz C. Ultrasonic separation of suspended particles-Part III: Application in biotechnology. Acustica. 1997;84:815-822

3. Brandt EH. Acoustic physics-Suspended by sound. Nature. 2001; 413:474-475.

4. Saito M, Kitamura N, Terauchi M. Ultrasonic manipulation of locomotive microorganisms and evaluation of their activity. $J$ Appl Phys. 2002;92:7581-7586.

5. Coakley WT. Ultrasonic separations in analytical biotechnology. Trends Biotechnol. 1997;15:506-511.
6. Thomas NE, Sobanski MA, Coakley WT. Ultrasonic enhancement of coated particle agglutination immunoassays: Influence of particle density and compressibility. Ultrasound Med Biol. 1999;25:443-450.

7. Gupta S, Feke DL. Filtration of particulate suspensions in acoustically driven porous media. AIChE J. 1998;44:1005-1014.

8. Elimelech M, Gregory J, Jia X, Williams RA. Particle Deposition and Aggregation-Measurement, Modeling and Simulation. Oxford, UK: Elsevier; 1995.

9. Grossner MT, Penrod AE, Belovich JM, Feke DL. Single-fiber model of particle retention in an acoustically driven porous mesh. Ultrasonics. 2003;41:65-74.

10. Gupta S, Feke DL. Acoustically driven collection of suspended particles within porous media. Ultrasonics. 1997;35:131-139.

11. Hill DH, Feke DL. Operating characteristics of acoustically driven filtration processes for particulate suspensions. Sep Sci Technol. 2000; 35:1363-1375.

12. Schwarz SE, Oldham WG. Electrical Engineering: An Introduction New York, NY: Oxford Univ. Press; 1993

13. Rusinko D. Design and Optimization of a Standing Wave Chamber. MS Thesis. Cleveland, OH: Case Western Reserve University; 2001. 